\title{
Not every irreversible airflow obstruction is COPD
}

\author{
José Baddini-Martinez ${ }^{1}$
}

\section{TO THE EDITOR}

The incorporation of clear concepts and the use of precise terminology are fundamental requirements to ensure good communication among health professionals as well as proper care of patients.

Airway diseases have always been fertile ground for the use of incorrect terms and the proliferation of nebulous ideas. Although it was to be expected that these problems would be reduced over time, conceptual vices remain, especially among physicians who are unfamiliar with our specialty.

A few years ago, I mentioned the risk of interpreting all wheezing as being due to bronchial asthma. ${ }^{(1)}$ Now I want to discuss a phenomenon seen particularly among general practitioners, residents in clinical medicine, and medical students: the belief that all irreversible airflow obstruction on spirometry implies the diagnosis of COPD.

The term COPD was coined more than five decades ago as being a pathophysiological change associated with chronic tobacco use. At that same time, emphysema was defined as a pathological concept involving destruction of distal airspaces. In contrast, chronic bronchitis was defined as a clinical change associated with having had cough and expectoration for a certain period of time.

As a consequence, the current definition recommended by the Global Initiative for Chronic Obstructive Lung Disease states that "COPD is a common preventable and treatable disease, characterized by persistent airflow limitation that is usually progressive and associated with enhanced chronic inflammatory responses in the airways and the lungs to noxious particles or gases. Exacerbations and comorbidities contribute to the overall severity in individual patients." ${ }^{\prime(3)}$

Therefore, the diagnosis of COPD necessarily requires findings of persistent airway obstruction on spirometry. Persistent or irreversible airflow obstruction is defined as obstruction that does not disappear or completely resolve after inhaled bronchodilator use. In this context, many patients with COPD can certainly respond to inhaled bronchodilators, but without a complete normalization of the $\mathrm{FEV}_{1} / \mathrm{FVC}$ ratio.

The second necessary element for the diagnosis of COPD is disease development in response to prolonged inhalation of noxious particles or gases. Although smoking is still the most important toxic element, it has been found in recent decades that other environmental exposures, especially to biomass burning, can also cause this disease. Thus, it is not acceptable to consider a diagnosis of COPD in patients without a consistent history of environmental exposure. In addition, it is important to emphasize that the expression "noxious particles or gases" refers to inert environmental exposures rather than to infectious agents. Therefore, obstructive lung disease due to sequelae of tuberculosis is not COPD.

In contrast, the diagnosis of asthma is based on the presence of dyspnea, wheezing, chest tightness, and cough. ${ }^{(4)}$ These symptoms vary in intensity over time, as does the degree of expiratory airflow obstruction. Similar to what happens in COPD, what determines the symptoms and the variable airflow obstruction is the presence of airway inflammation. However, this inflammation is distinct in character, being generally eosinophilic in nature, as opposed to that of COPD, which is usually neutrophilic. In addition, what causes asthma, at least at onset, are mechanisms of atopy and hypersensitivity to inhaled agents. Being primarily immunoallergic in nature, these reactions do not have a close relationship with the duration of exposure or with the concentration of inhaled material, unlike COPD. Asthma generally begins in childhood, has a strong genetic influence, and is usually accompanied by other signs of atopy. In addition to the fact that COPD is a disease whose symptoms appear later in life, the exposures related to the development of COPD do not involve sensitization mechanisms and are distinct from those associated with the development of asthma: wood stove smoke vs. cockroach antigens, for example.

The crucial point that I want to raise here is the following: in the same way that confirming the diagnosis of COPD requires findings of irreversible airflow obstruction on spirometry, raising the diagnostic possibility of COPD requires the identification in the patient's history of environmental exposure of findings suggestive of, for example, smoking or exposure to smoke from biomass burning for home heating and cooking purposes. The only exception to this rule would be emphysema caused by alpha- 1 antitrypsin deficiency. However, this is a rare condition, in which exposure to environmental smoke often also occurs. For this reason, in every patient under 45 years of age who is suspected of having COPD, serum levels of alpha- 1 antitrypsin should be determined.

Obviously, when faced with any lung disease, anamnesis takes on importance for characterizing not only current symptoms and their triggering agents but also the occurrence of previous respiratory symptoms, occupational history, family history, etc. These data, together with physical examination and standard anteroposterior and lateral X-rays of the chest, provide the foundation for the differential diagnosis of various lung diseases. 
Chart 1 lists various clinical conditions that can result in persistent airflow obstruction on simple spirometry but do not meet the diagnosis of COPD because of the lack of appropriate environmental exposure as a factor associated with their development. It is important to emphasize that all of the listed conditions can, more or less frequently, produce an isolated airflow obstruction pattern on spirometry. That is, more complex issues involving a reduced or very reduced FVC on spirometry, which can be classified as a nonspecific or mixed pattern, are not being discussed here. Certainly, in these two last conditions, complete pulmonary function studies, involving measurements of TLC and RV, are ideal for the proper characterization of the functional diagnosis and, consequently, of the underlying lung disease itself. In fact, the finding of a nonspecific mixed pattern in patients in whom COPD is strongly suspected usually means the occurrence of some other superimposed comorbid condition. ${ }^{(5)}$

In summary, although COPD is a very common condition, whenever patients have a persistent airflow
Chart 1. Some causes of persistent pulmonary obstruction other than COPD.

Severe asthma
Bronchiectasis
Tracheal stenosis or airway stenosis: after intubation;
presence of tumors or foreign bodies; infectious
stenosis; or postinfectious stenosis (tuberculosis,
paracoccidioidomycosis, etc.)
Sarcoidosis
Lymphangioleiomyomatosis
Langerhans cell histiocytosis
Bronchiolitis obliterans

obstruction pattern on spirometry but no consistent history of environmental exposure, they should undergo further testing, particularly flow-volume curves and pulmonary function testing (including measurements of TLC and DLCO), as well as HRCT scanning of the chest. So, in a nutshell, not every irreversible airflow obstruction is COPD!

\section{REFERENCES}

1. Martinez JA. Not all that wheezes is asthma! J Bras Pneumol 2013:39(4):518-20. http://dx.doi.org/10.1590/S1806-37132013000400017

2. Terminology, definitions and classifications of chronic pulmonary emphysema and related conditions: a report of the conclusions of a Ciba Guest Symposium. Thorax. 1959;14(4):286-99. http://dx.doi. org/10.1136/thx.14.4.286

3. Global Initiative for Chronic Obstructive Lung Disease - GOLD [homepage on the Internet]. Bethesda: Global Initiative for Chronic Obstructive Lung Disease [cited 2016 Jun 24]. Global Strategy for the Diagnosis, Management, and Prevention of COPD 2016. Available from: http://goldcopd.org/global-strategy-diagnosis-managementprevention-copd-2016/

4. Reddel HK, Bateman ED, Becker A, Boulet LP, Cruz AA, Drazen $\mathrm{JM}$, et al. A summary of the new GINA strategy: a roadmap to asthma control. Eur Respir J. 2015;46(3):622-39. http://dx.doi. org/10.1183/13993003.00853-2015

5. Wan ES, Hokanson JE, Murphy JR, Regan EA, Make BJ, Lynch DA, et al. Clinical and radiographic predictors of GOLD-unclassified smokers in the COPDGene study. Am J Respir Crit Care Med. 2011;184(1):57-63. http://dx.doi.org/10.1164/rccm.201101-00210C 